\title{
Cognitive behavioral therapy for suicidal behaviors: improving patient outcomes
}

This article was published in the following Dove Press journal:

Psychology Research and Behavior Management

3 March 2016

Number of times this article has been viewed

\section{Louise Mewton'}

Gavin Andrews ${ }^{2}$

'National Health and Medical Research Council Centre for Research Excellence in Mental Health and Substance Use, National Drug and Alcohol Research Centre, University of New South Wales, ${ }^{2}$ Clinical Research Unit for Anxiety and Depression (CRUfAD), St Vincent's Hospital, Sydney, NSW, Australia
Correspondence: Louise Mewton National Health and Medical Research

Council Centre for Research Excellence in Mental Health and Substance Use, National Drug and Alcohol Research Centre, University of New South Wales, Sydney, NSW, 2052 Australia

Tel +6I 28936 II 3 |

Email louisem@unsw.edu.au
Abstract: This systematic review provides an overview of the effectiveness of cognitive behavioral therapy (CBT) in reducing suicidal cognitions and behavior in the adult population. We identified 15 randomized controlled trials of CBT for adults (aged 18 years and older) that included suicide-related cognitions or behaviors as an outcome measure. The studies were identified from PsycINFO searches, reference lists, and a publicly available database of psychosocial interventions for suicidal behaviors. This review identified some evidence of the use of CBT in the reduction of both suicidal cognitions and behaviors. There was not enough evidence from clinical trials to suggest that CBT focusing on mental illness reduces suicidal cognitions and behaviors. On the other hand, CBT focusing on suicidal cognitions and behaviors was found to be effective. Given the current evidence, clinicians should be trained in CBT techniques focusing on suicidal cognitions and behaviors that are independent of the treatment of mental illness.

Keywords: suicidal behaviors, suicidal cognitions, CBT

\section{Introduction}

Internationally, it is estimated that 800,000 people die by suicide each year. Suicide is the 15 th leading cause of death worldwide, accounting for $1.4 \%$ of all deaths, and the second leading cause of death among 15-29 year olds globally. ${ }^{1}$ In the period between 2000 and 2012, suicide rates dropped by 9\% internationally. ${ }^{1}$ This statistic masks considerable cross-national differences, but indicates a global trend toward a reduction in suicide. Suicide prevention strategies are therefore having some impact on the suicide rate. To date, there is good evidence to suggest that universal prevention strategies that target entire populations, such as the restriction of access to lethal means, are effective in reducing suicide rates. ${ }^{2}$ Such strategies have become central to national responses to suicide prevention. To further reduce suicide deaths and improve patient outcomes, these universal strategies need to be supplemented with selective or indicated prevention strategies that target groups or individuals at high risk for suicide.

Such targeted interventions may focus on individuals seeking treatment for mental illness. Mental illness is one of the strongest risk factors for suicide, having been implicated in $91 \%$ of completed suicides. ${ }^{3}$ The risk of suicide is highest among those with borderline personality disorder, followed by depression, bipolar disorder, opioid use, and schizophrenia. ${ }^{4}$ It is estimated that between half- and three-quarters of all suicides would be avoided if mental illness were eradicated. ${ }^{3}$ Similar population attributable risk estimates exist between smoking and lung cancer. ${ }^{5}$ Recent reviews have shown that antipsychotics reduce the risk of suicide in people with schizophrenia ${ }^{6}$ and that lithium is an effective 
treatment for reducing the risk of suicide in people with mood disorders, and bipolar disorder in particular. ${ }^{7}$ Whether antidepressants reduce suicide risk is debatable, ${ }^{8-10}$ perhaps due to the frequent exclusion of actively suicidal participants from antidepressant trials. ${ }^{11,12}$ Psychotherapies, including cognitive behavioral therapy (CBT) and dialectical behavior therapy (DBT), have not been shown to improve suicidal outcomes when mental illness is the main treatment focus. ${ }^{13}$

On the other hand, both CBT and DBT have been shown to be effective in reducing suicidal risk when these treatments focus on suicidal cognitions and behaviors rather than mental illness. ${ }^{13,14}$ This suggests that better patient outcomes may arise when psychotherapy focuses on suicidal cognitions and behaviors as dysfunctional individual factors rather than symptoms of mental illness. ${ }^{15}$ Alternatively, the discrepant findings regarding psychotherapies tailored toward either mental illness or suicidality may also be due to the lack of power and inadequately designed studies. Generally, randomized controlled trials of psychotherapies for mental illness do not systematically assess suicidal behaviors as an outcome measure, ${ }^{2}$ resulting in a poor evidence base. A recent review of 1,344 studies focusing on psychotherapy for depression only found three studies that reported outcomes related to suicidal cognitions or behaviors. ${ }^{16}$ Over recent years, the assessment of suicidal cognitions and behaviors in studies of psychotherapies has improved. A publicly available database focusing on psychosocial interventions in general for suicidal ideation, plans, and attempts ${ }^{17}$ recently identified 154 randomized controlled trials that assessed suicidal cognitions or behaviors as an outcome measure.

\section{The current review}

This review aims to provide an overview of the effectiveness of CBT, specifically in reducing suicidal activity in the adult population. CBT is an umbrella term for various treatments that focus on challenging cognitive biases (through cognitive restructuring) and behaviors (through graded exposure and relaxation training). Given the therapeutic focus of CBT, this review will focus on studies that report either suicidal cognitions (ie, suicidal ideation) or suicidal behaviors (ie, deliberate self-harm and suicidal attempts) as outcome measures. Suicidal cognitions and behaviors clearly exist along a continuum of severity, and only a minority of individuals will progress from suicidal ideation and self-harm through to suicide attempts. ${ }^{18}$ Regardless of severity, these cognitions and behaviors confer a degree of suicide risk and constitute targets for treatment in their own right. ${ }^{13}$

In order to reduce heterogeneity and provide a more focused discussion, this review will exclude other psychosocial interventions and therapies, such as DBT, which include additional therapeutic strategies and components that limit comparability to standard CBT. Again to reduce the heterogeneity of the included studies, the scope of the present review will be limited to adults only (ie, those aged 18 years and older). Several recent reviews have focused on therapeutic interventions for suicidal cognitions and behaviors in adolescents. ${ }^{19-24}$ These reviews provide an up-to-date overview of the literature and also serve to highlight the developmental, clinical, and social issues that limit comparisons between adolescent and adult populations when considering suicidal outcomes.

The studies included in this review were identified via the PsycINFO database, which was searched from 1980 to August 2015 using the following search terms adapted from a previous review conducted by Tarrier et al13: "suicid* AND therapy AND cognitive OR behavio*ral", "CBT AND suicid*”, "CBT AND self-harm OR self-injury", "CBT AND suicidal behavio*r", "suicide* AND intervention", and "suicide* AND therapy". Only randomized controlled trials that compared CBT with a control group were included. Studies needed to be reported in English in a peer-reviewed journal, and suicidal cognitions and/or behaviors needed to be included as an outcome measure. Studies that included composite measures that could not be disaggregated into outcomes reflecting either suicidal cognitions or suicidal behaviors were excluded. Case studies, reviews, clinical descriptions, and discussion articles were excluded, as were pilot and feasibility studies. A total of 3,176 articles were identified through PsycINFO, and the titles and abstracts of these articles were inspected. Reference lists of relevant reviews and key articles were also inspected, as was the publicly available database of 154 psychosocial interventions mentioned earlier. ${ }^{17}$

A total of 15 studies met the inclusion criteria and were included in this review. An overview of the findings from these studies is listed in Tables 1 and 2. All studies reported in Tables 1 and 2 conducted the randomized controlled trials of CBT (including psychoeducation, applied relaxation, and graded exposure) for adults (aged 18 years and older) and included suicidal cognitions and/or behaviors as an outcome measure. Any suicidal outcomes reported during the active treatment phase were not included in Tables 1 and 2. In order to facilitate comparisons and synthesize the study findings, Tables 1 and 2 also include an effect size estimate (in most cases, Cohen's $d$ ) for each of the included studies. Several of these studies did not report effect sizes and related confidence intervals, and these were calculated instead from the available data using ClinTools Software. ${ }^{25}$ For continuous 


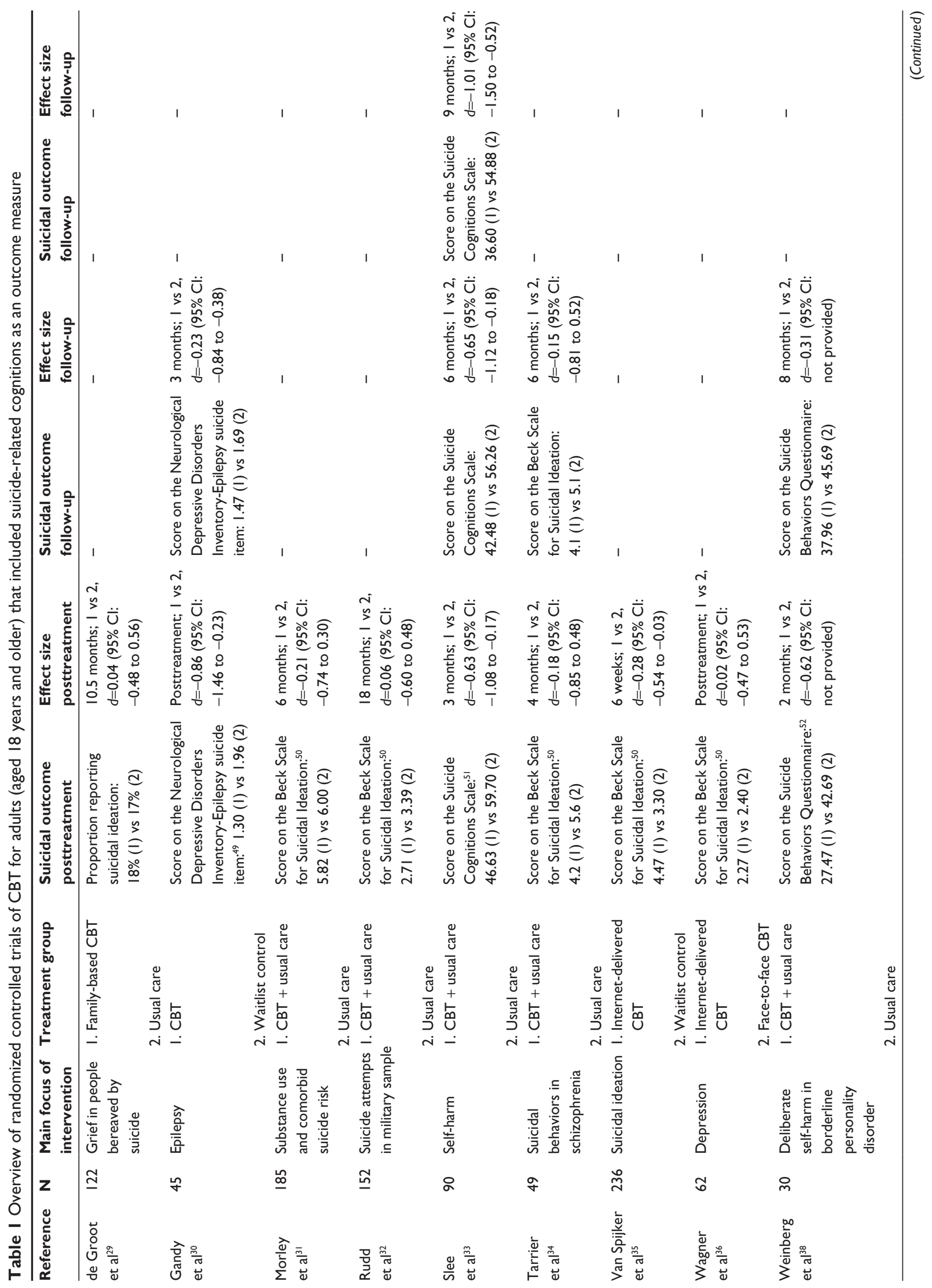




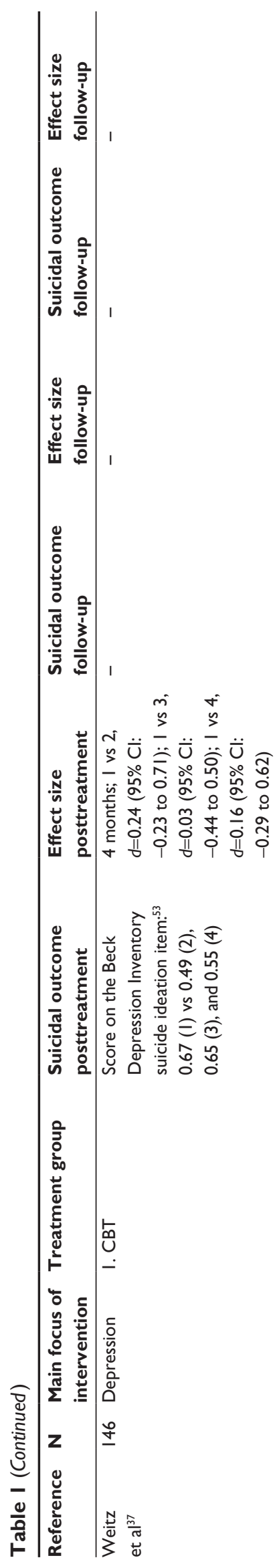

variables, Cohen's $d$ and related confidence intervals were calculated using the posttreatment or follow-up assessment means and pooled standard deviations. In cases where odds ratios and proportions were provided rather than means and standard deviations, Cohen's $d$ was calculated using the standard formulae implemented in the ClinTools Software. In cases where the necessary data were not available in the original article, we contacted the corresponding author and requested the unpublished data. We were unable to source the data necessary for calculating effect sizes for one additional study, ${ }^{26}$ which was subsequently excluded from this review. Negative effect sizes presented in Tables 1 and 2 indicate an effect in favor of CBT. In the following discussion, these effect sizes are interpreted in relation to established criteria for trivial $(< \pm 0.20)$, small $(\geq \pm 0.20)$, medium $(\geq \pm 0.50)$, and large $(\geq \pm 0.80)$ effect sizes. ${ }^{27}$ Where data were available, forest plots depicting the study effect sizes and confidence intervals were also constructed using SAS Version 9.2. ${ }^{28}$

\section{Overview of findings}

Ten of the 15 included studies measured suicidal cognitions, ${ }^{29-38}$ and there were a total of 17 comparisons with suicidal cognitions as the outcome of interest (Table 1). Seven of these comparisons focusing on cognitions elicited effect sizes that were trivial according to the established criteria. Four of these comparisons focusing on cognitions demonstrated small effect sizes in favor of CBT, and one study focusing on cognitions demonstrated a small effect size in favor of interpersonal therapy over CBT. Finally, when suicidal cognitions were the outcome of interest, there were four medium effect sizes and one large effect size in favor of CBT. The effect sizes for suicidal cognitions at the first assessment posttreatment are plotted in Figure 1. Only studies with both an effect size and an associated confidence interval at posttreatment in Table 1 are included in Figure 1.

Eight of the 15 included studies measured suicidal behaviors, ${ }^{32,33,38-43}$ and there were a total of 14 comparisons with suicidal behaviors as the outcome of interest (Table 2). Five of these comparisons focusing on behaviors demonstrated trivial effect sizes. Six studies focusing on behaviors elicited small effect sizes in favor of CBT, and one study focusing on behaviors elicited a small effect size in favor of usual care over CBT. Finally, when suicidal behaviors were the outcome of interest, there were three medium effect sizes in favor of CBT. The effect sizes for suicidal behaviors at the first assessment posttreatment are plotted in Figure 2. Only studies with both an effect size and 


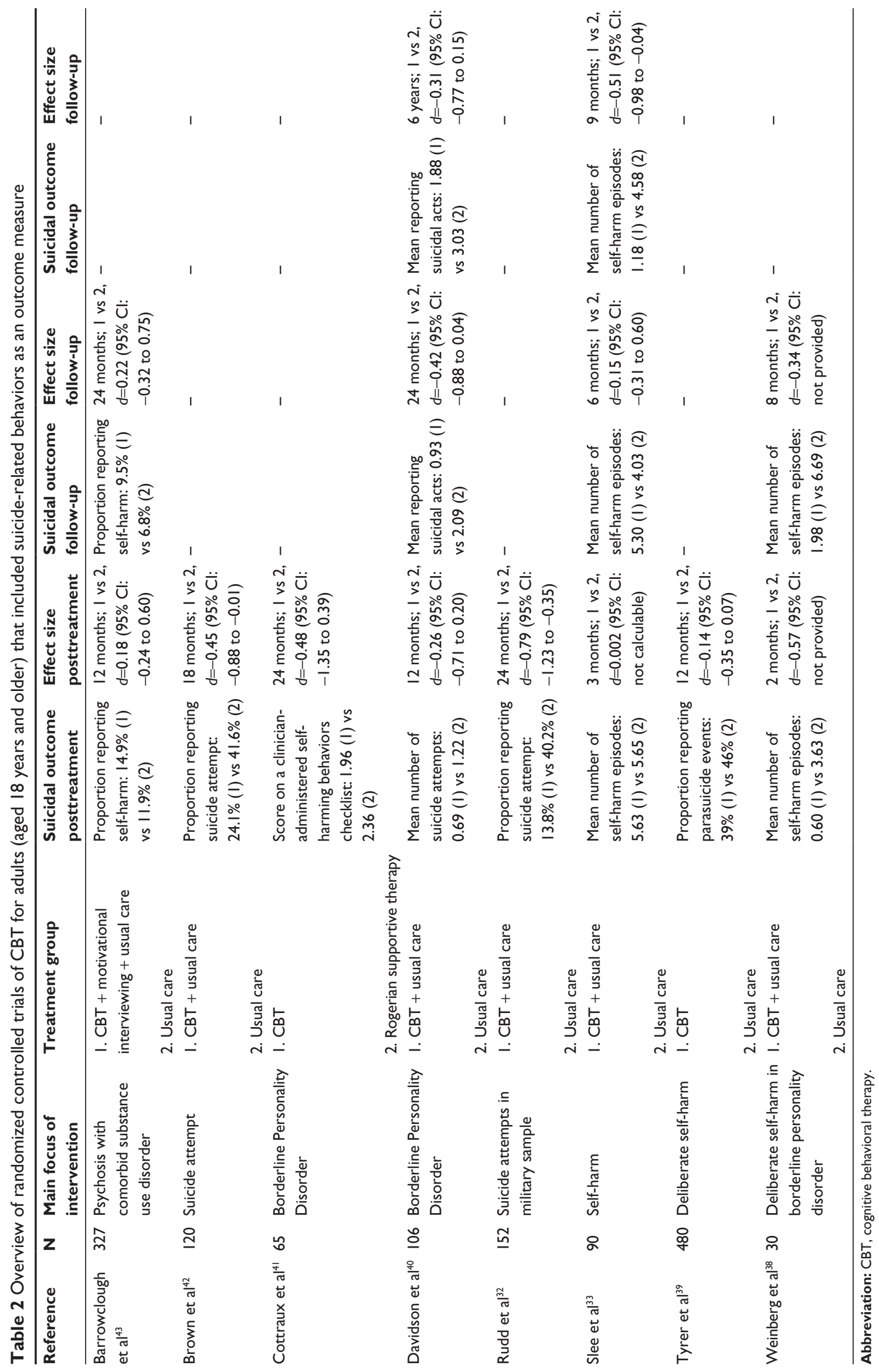




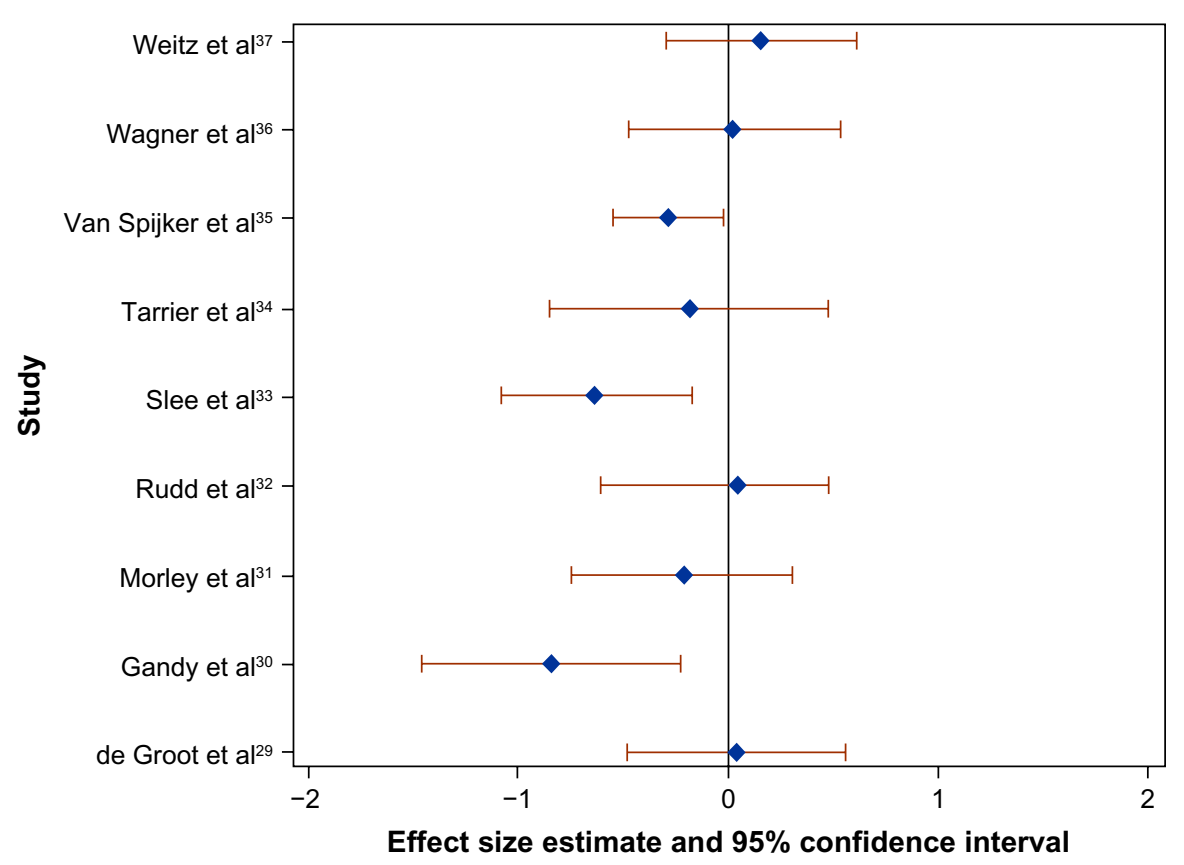

Figure I Forest plot of the effect size (Cohen's $d$ ) of CBT on suicidal cognitions compared to the control group at the first assessment posttreatment in nine randomized controlled trials.

Note: Only studies with both an effect size and associated confidence interval at posttreatment were included in this plot.

Abbreviation: CBT, cognitive behavioral therapy.

associated confidence interval at posttreatment in Table 2 Two studies focused on depression, ${ }^{36,37}$ and two studies are included in Figure 2.

\section{Intervention focus}

Seven of the 15 included studies focused on suicidal cognitions or behaviors as the main intervention focus. ${ }^{32-35,38,39,42}$ focused on borderline personality disorder. ${ }^{40,41}$ The treatment focus of one study was psychosis with comorbid substance abuse, ${ }^{43}$ while for another study the focus was substance use with comorbid suicide risk. ${ }^{31}$ Two further studies focused on epilepsy ${ }^{30}$ and grief in people bereaved by suicide. ${ }^{29}$ The

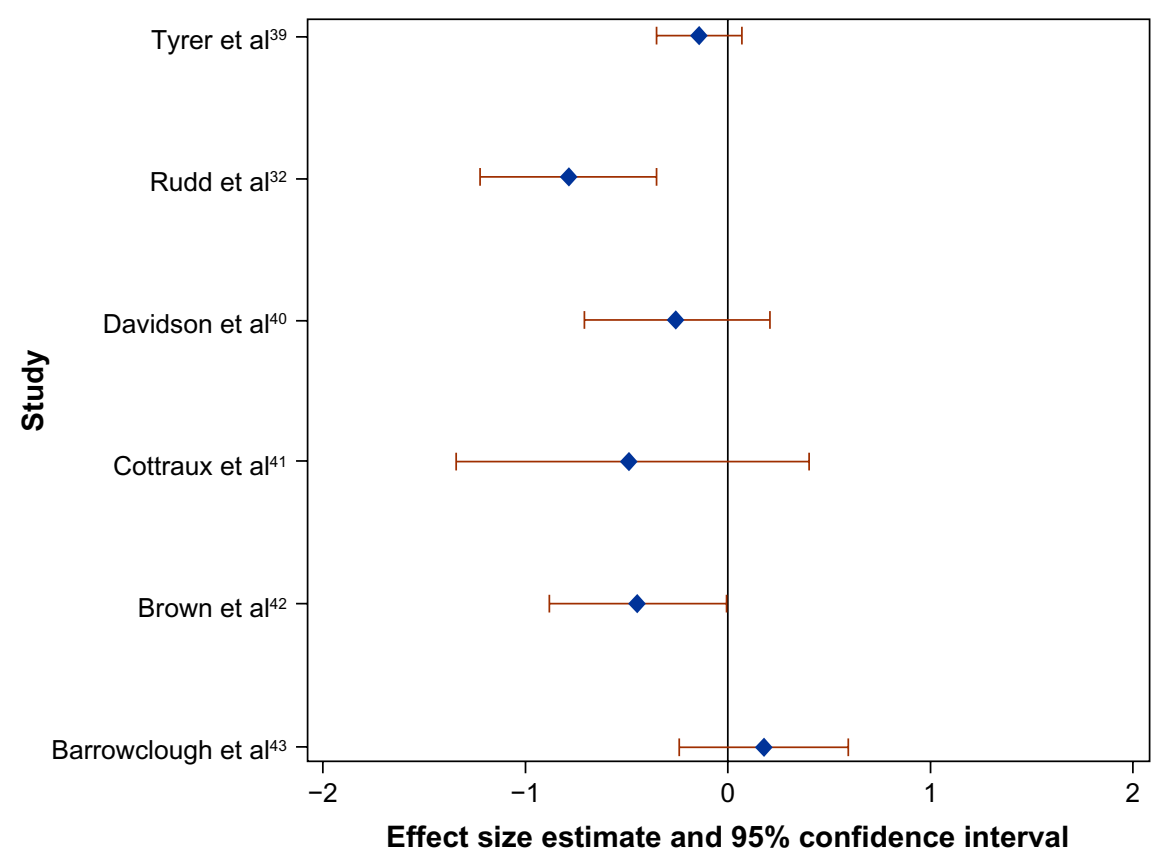

Figure 2 Forest plot of the effect size (Cohen's $d$ ) of CBT on suicidal behaviors compared to the control group at the first assessment posttreatment in six randomized controlled trials.

Note: Only studies with both an effect size and associated confidence interval at posttreatment were included in this plot.

Abbreviation: CBT, cognitive behavioral therapy. 
strongest effect sizes were elicited from interventions that focused specifically on suicidal behaviors or cognitions. The two treatments focusing on borderline personality disorder demonstrated consistent small effects in favor of CBT. ${ }^{40,41}$ For one treatment focusing on depression, CBT was not found to be more effective than an antidepressant or placebo pill. The same study demonstrated a small effect size in favor of interpersonal therapy for depression over CBT. ${ }^{37}$ The other study focusing on depression found that suicidal outcomes did not differ between internet-delivered CBT and face-toface CBT. ${ }^{36}$

\section{Control group}

Three of the 15 studies included active control groups, while the remaining 12 studies used a usual care or waitlist control group. When focusing on CBT for borderline personality disorder, there was a small effect size in favor of CBT over Rogerian supportive therapy in the reduction of suicidal behaviors. ${ }^{41}$ There were no differences between Internet-delivered CBT and face-to-face CBT for depression in reducing suicidal cognitions. ${ }^{36}$ However, when examining change in suicidal cognitions within each group, the study authors only found a statistically significant change in the face-to-face CBT group from baseline to follow-up. One final study focusing on depression compared CBT with interpersonal therapy, imipramine, and placebo. ${ }^{37}$ When focusing on suicidal cognitions, there was a small effect size in favor of interpersonal therapy over CBT, whereas the effect size differences between CBT, imipramine, and placebo were trivial.

\section{Longer term follow-up}

None of the studies measuring suicidal cognitions reported outcomes extending $>9$ months. Four studies focusing on suicidal cognitions included multiple assessment occasions. In one study, a trivial effect size persisted over 2-month and 6-month follow-up occasions, ${ }^{34}$ whereas two studies demonstrated diminishing effect sizes over subsequent assessment occasions. ${ }^{30,38}$ In one study, however, the superiority of CBT over usual care in terms of suicidal behaviors was only evident in later assessment occasions. ${ }^{33}$ This same study also measured suicidal cognitions as an outcome and similarly elicited larger effect sizes in later assessment occasions. Otherwise, effect sizes either remained stable ${ }^{40,43}$ or diminished ${ }^{38}$ across assessment occasions when suicidal behaviors were the outcome of interest. One study focusing on suicide attempts demonstrated persistent small effect sizes at 1 year, 2 years, and 6 years posttreatment. ${ }^{40}$ Another elicited a medium effect size at 2 years posttreatment. ${ }^{32}$
Two other studies assessed deliberate self-harm at 2 years posttreatment. One study elicited a small effect size in favor of $\mathrm{CBT},{ }^{41}$ whereas the other study elicited small effect size in favor of usual care. ${ }^{43}$

\section{Discussion}

This systematic review identified 15 studies that focused on either suicidal cognitions or suicidal behaviors as outcomes for CBT. This review focused specifically on cognitions and behaviors because these are the central elements that CBT seeks to change. This review identified evidence for the use of CBT in the reduction of both suicidal cognitions and behaviors. CBT is therefore a useful strategy in the prevention of suicidal cognitions and suicidal behaviors.

CBT has been shown to be effective in reducing the symptoms of mental illnesses that are associated with an increased risk of suicide, including depression, anxiety, and psychosis. ${ }^{44}$ Large clinical reports have also shown that suicidal cognitions and behaviors reduce significantly following CBT for depression. ${ }^{45,46}$ At present, however, there is not enough evidence from clinical trials to suggest that CBT focusing on these illnesses also reduces suicidal cognitions and behaviors. This is consistent with previous reviews of the literature. ${ }^{13,14}$ Mental illness is implicated in most suicides, ${ }^{3}$ and the risk of suicide among individuals suffering from a mental illness is many times greater than that of the general population. ${ }^{4}$ Given this evidence, the effective prevention and treatment of mental illness should be a central component of suicide prevention strategies. This review highlights the need to better evaluate the extent to which interventions such as CBT, which has been shown to be effective in treating a range of mental illnesses, also reduce suicidal cognitions and behaviors. Clinical trials may be constrained by ethical concerns over randomizing actively suicidal individuals to an inactive treatment arm. Following strict and comprehensive safety assessment and management procedures, such as those reported in the study by Brown et al, ${ }^{42}$ should counteract these concerns. Innovative methods in the design of clinical trials, such as those informed by sequential ${ }^{47}$ or platform ${ }^{48}$ methods, should also be adopted to optimize patient safety and reduce inactive treatment length.

\section{Limitations}

This review needs to be considered within the context of some limitations. A formal methodological quality evaluation was not undertaken for this review, but there are methodological problems observed in the primary studies that limit the conclusions of this review. For example, the sample sizes in the primary studies were occasionally small, and the 
representativeness of the study participants is often limited. We attempted to reduce the heterogeneity of the included studies, but the comparability across studies was ultimately low. This review covers treatments designed to address a variety of phenomena, and this is evident in the diversity of treatment components and associated clinical methods. Furthermore, inconsistencies in assessment measures and timing of assessment occasions limited the cross-study comparisons. Primary and secondary endpoints were uniquely defined for each study, making it difficult to combine the data and assess the short-, medium-, and long-term effectiveness of CBT on suicidality. It is also not clear whether the studies included in this review represent the full spectrum of severity with regards to suicidal cognitions and behaviors. Six of the studies in this review excluded participants with severe suicidal cognitions and/or behaviors, ${ }^{30,34-38}$ and mean posttreatment scores on continuous measures of suicidal cognitions were often low even in inactive control groups. A restricted range of severity is likely to have some impact on the between-group effect sizes reported in this review, and may explain some of the small or trivial effect sizes generated. Finally, the suicidal outcomes assessed in these studies did not include completed suicide. The extent to which reducing suicidal cognitions and behaviors actually prevents completed suicide is unknown. It can only be assumed that reducing suicidal ideation, self-harm, and suicide attempts will have some, albeit unknown, effect on suicide rates.

This review focused on CBT, a psychotherapy known to be highly efficacious in the reduction of symptoms related to a range of mental illnesses. ${ }^{44} \mathrm{CBT}$ is a useful strategy for reducing suicidal cognitions and behaviors. In terms of CBT targeting mental illnesses, there is currently insufficient evidence to evaluate its effectiveness in reducing suicidal outcomes. Given the current evidence, clinicians should be trained in CBT techniques focusing on suicidal cognitions and behaviors that are independent of the treatment of mental illness.

\section{Disclosure}

The authors report no conflicts of interest in this work.

\section{References}

1. World Health Organisation [webpage on the Internet]. Preventing Suicide: A Global Imperative. Key Messages. Available from: www. who.int/mental_health/suicide-prevention/key_messages.pdf. Accessed October 23, 2015.

2. Mann JJ, Apter A, Bertolote J, et al. Suicide prevention strategies: a systematic review. JAMA. 2005;294(16):2064-2074.

3. Cavanagh JT, Carson AJ, Sharpe M, Lawrie SM. Psychological autopsy studies of suicide: a systematic review. Psychol Med. 2003;33(03):395-405.
4. Chesney E, Goodwin GM, Fazel S. Risks of all-cause and suicide mortality in mental disorders: a meta-review. World Psychiatry. 2014;13(2):153-160.

5. Goldney RD, Dal Grande E, Fisher LJ, Wilson D. Population attributable risk of major depression for suicidal ideation in a random and representative community sample. J Affect Disord. 2003;74(3): 267-272.

6. Reutfors J, Bahmanyar S, Jönsson EG, et al. Medication and suicide risk in schizophrenia: a nested case-control study. Schizophr Res. 2013;150(2):416-420.

7. Cipriani A, Hawton K, Stockton S, Geddes JR. Lithium in the prevention of suicide in mood disorders: updated systematic review and metaanalysis. BMJ. 2013;346:f3646.

8. Khan A, Khan S, Kolts R, Brown WA. Suicide rates in clinical trials of SSRIs, other antidepressants, and placebo: analysis of FDA reports. Am J Psychiatry. 2003;160(4):790-792.

9. Gunnell D, Saperia J, Ashby D. Selective serotonin reuptake inhibitors (SSRIs) and suicide in adults: meta-analysis of drug company data from placebo controlled, randomised controlled trials submitted to the MHRA's safety review. BMJ. 2005;330(7488):385.

10. Fergusson D, Doucette S, Glass KC, et al. Association between suicide attempts and selective serotonin reuptake inhibitors: systematic review of randomised controlled trials. BMJ. 2005;330(7488):396.

11. Zimmerman M, Chelminski I, Posternak MA. Generalizability of antidepressant efficacy trials: differences between depressed psychiatric outpatients who would or would not qualify for an efficacy trial. $A m J$ Psychiatry. 2005;162(7):1370-1372.

12. Van der Lem R, van der Wee N, van Veen T, Zitman F. The generalizability of antidepressant efficacy trials to routine psychiatric out-patient practice. Psychol Med. 2011;41(07):1353-1363.

13. Tarrier N, Taylor K, Gooding P. Cognitive-behavioral interventions to reduce suicide behavior a systematic review and meta-analysis. Behav Modif. 2008;32(1):77-108.

14. Comtois KA, Linehan MM. Psychosocial treatments of suicidal behaviors: a practice-friendly review. J Clin Psychol. 2006;62(2): 161-170.

15. Linehan MM. Suicide intervention research: a field in desperate need of development. Suicide Life Threat Behav. 2008;38(5):483-485.

16. Cuijpers P, de Beurs DP, van Spijker BA, Berking M, Andersson G, Kerkhof AJ. The effects of psychotherapy for adult depression on suicidality and hopelessness: a systematic review and meta-analysis. J Affect Disord. 2013;144(3):183-190.

17. Christensen H, Calear AL, Van Spijker B, et al. Psychosocial interventions for suicidal ideation, plans, and attempts: a database of randomised controlled trials. BMC Psychiatry. 2014;14(1):86.

18. Kessler RC, Borges G, Walters EE. Prevalence of and risk factors for lifetime suicide attempts in the National Comorbidity Survey. Arch Gen Psychiatry. 1999;56(7):617-626.

19. Ougrin D, Tranah T, Stahl D, Moran P, Asarnow JR. Therapeutic interventions for suicide attempts and self-harm in adolescents: systematic review and meta-analysis. J Am Acad Child Adolesc Psychiatry. 2015;54(2):97-107.e2.

20. Brent DA, McMakin DL, Kennard BD, Goldstein TR, Mayes TL, Douaihy AB. Protecting adolescents from self-harm: a critical review of intervention studies. $J$ Am Acad Child Adolesc Psychiatry. 2013;52(12):1260-1271.

21. Corcoran J, Dattalo P, Crowley M, Brown E, Grindle L. A systematic review of psychosocial interventions for suicidal adolescents. Child Youth Serv Rev. 2011;33(11):2112-2118.

22. King CA, Merchant CR. Social and interpersonal factors relating to adolescent suicidality: a review of the literature. Arch Suicide Res. 2008;12(3):181-196.

23. Robinson J, Hetrick SE, Martin C. Preventing suicide in young people: systematic review. Aust N Z J Psychiatry. 2011;45(1):3-26.

24. Robinson J, Cox G, Malone A, et al. A systematic review of schoolbased interventions aimed at preventing, treating, and responding to suicide-related behavior in young people. Crisis. 2013;34(3): 164-182. 
25. Devilly GJ. ClinTools Software for Windows: Version 4.0 (Computer Programme). Hawthorn, VIC: Brain Sciences Institute, Swinburne University; 2005.

26. Blum N, St John D, Pfohl B, et al. Systems training for emotional predictability and problem solving (STEPPS) for outpatients with borderline personality disorder: a randomized controlled trial and 1-year follow-up. Am J Psychiatry. 2008;165(4):468-478.

27. Cohen J. A power primer. Psychol Bull. 1992;112(1):155.

28. SAS Institute Inc. SAS version 9.2. Cary, NC: SAS Intsitute Inc; 2008.

29. de Groot M, de Keijser J, Neeleman J, Kerkhof A, Nolen W, Burger H. Cognitive behaviour therapy to prevent complicated grief among relatives and spouses bereaved by suicide: cluster randomised controlled trial. BMJ. 2007;334(7601):994.

30. Gandy M, Sharpe L, Nicholson Perry K, et al. Cognitive behaviour therapy to improve mood in people with epilepsy: a randomised controlled trial. Cogn Behav Ther. 2014;43(2):153-166.

31. Morley KC, Sitharthan G, Haber PS, Tucker P, Sitharthan T. The efficacy of an opportunistic cognitive behavioral intervention package (OCB) on substance use and comorbid suicide risk: a multisite randomized controlled trial. J Consult Clin Psychol. 2014;82(1):130-140.

32. Rudd M, Bryan CJ, Wertenberger EG, et al. Brief cognitive-behavioral therapy effects on post-treatment suicide attempts in a military sample: results of a randomized clinical trial with 2-year follow-up. Am J Psychiatry. 2015;172(5):441-449.

33. Slee N, Garnefski N, van der Leeden R, Arensman E, Spinhoven P. Cognitive-behavioural intervention for self-harm: randomised controlled trial. Br J Psychiatry. 2008;192(3):202-211.

34. Tarrier N, Kelly J, Maqsood S, et al. The cognitive behavioural prevention of suicide in psychosis: a clinical trial. Schizophr Res. 2014;156(2-3):204-210.

35. van Spijker BA, van Straten A, Kerkhof AJ. Effectiveness of online self-help for suicidal thoughts: results of a randomised controlled trial. PLoS One. 2014;9(2):e90118.

36. Wagner B, Horn AB, Maercker A. Internet-based versus face-to-face cognitive-behavioral intervention for depression: a randomized controlled non-inferiority trial. J Affect Disord. 2014;15(2-154):113-121.

37. Weitz E, Hollon SD, Kerkhof A, Cuijpers P. Do depression treatments reduce suicidal ideation? The effects of CBT, IPT, pharmacotherapy, and placebo on suicidality. J Affect Disord. 2014;167:98-103.

38. Weinberg I, Gunderson JG, Hennen J, Cutter CJ, Jr. Manual assisted cognitive treatment for deliberate self-harm in borderline personality disorder patients. J Pers Disord. 2006;20(5):482-492.

39. Tyrer P, Thompson S, Schmidt U, et al. Randomized controlled trial of brief cognitive behaviour therapy versus treatment as usual in recurrent deliberate self-harm: the POPMACT study. Psychol Med. 2003;33(6):969-976.
40. Davidson KM, Tyrer P, Norrie J, Palmer SJ, Tyrer H. Cognitive therapy v. usual treatment for borderline personality disorder: prospective 6-year follow-up. Br J Psychiatry. 2010;197(6):456-462.

41. Cottraux J, Boutitie F, Milliery M, et al. Cognitive therapy versus Rogerian supportive therapy in borderline personality disorder. Psychother Psychosom. 2009;78(5):307-316.

42. Brown GK, Have TT, Henriques GR, Xie SX, Hollander JE, Beck AT. Cognitive therapy for the prevention of suicide attempts: a randomized controlled trial. JAMA. 2005;294(5):563-570.

43. Barrowclough $\mathrm{C}$, Haddock $\mathrm{G}$, Wykes $\mathrm{T}$, et al. Integrated motivational interviewing and cognitive behavioural therapy for people with psychosis and comorbid substance misuse: randomised controlled trial. BMJ. 2010;341:c6325.

44. Butler AC, Chapman JE, Forman EM, Beck AT. The empirical status of cognitive-behavioral therapy: a review of meta-analyses. Clin Psychol Rev. 2006;26(1):17-31.

45. Mewton L, Andrews G. Cognitive behaviour therapy via the internet for depression: a useful strategy to reduce suicidal ideation. $J$ Affect Disord. 2015;170:78-84.

46. Watts S, Newby JM, Mewton L, Andrews G. A clinical audit of changes in suicide ideas with internet treatment for depression. BMJ Open. 2012;2(5):e001558.

47. Schäfer H, Müller HH. Construction of group sequential designs in clinical trials on the basis of detectable treatment differences. Stat Med. 2004;23(9):1413-1424.

48. Berry SM, Connor JT, Lewis RJ. The platform trial: an efficient strategy for evaluating multiple treatments. JAMA. 2015;313(16):1619-1620.

49. Gilliam FG, Barry JJ, Hermann BP, Meador KJ, Vahle V, Kanner AM Rapid detection of major depression in epilepsy: a multicentre study. Lancet Neurol. 2006;5(5):399-405.

50. Beck AT, Steer RA, Ranieri WF. Scale for suicide ideation: psychometric properties of a self-report version. J Clin Psychol. 1988; 44(4):499-505.

51. Bryan CJ, Rudd MD, Wertenberger E, Etienne N, Ray-Sannerud BN, Morrow CE, Peterson AL, Young-McCaughon S. Improving the detection and prediction of suicidal behavior among military personnel by measuring suicidal beliefs: An evaluation of the Suicide Cognitions Scale. J Affect Disord. 2014;159:1522.

52. Linehan MM, Nielsen SL. Assessment of suicide ideation and parasuicide: hopelessness and social desirability. J Consult Clin Psychol. 1981;49(5):773.

53. Beck AT, Steer RA, Carbin MG. Psychometric properties of the beck depression inventory: twenty-five years of evaluation. Clin Psychol Rev. 1988;8(1):77-100.

Psychology Research and Behavior Management

\section{Publish your work in this journal}

Psychology Research and Behavior Management is an international, peerreviewed, open access journal focusing on the science of psychology and its application in behavior management to develop improved outcomes in the clinical, educational, sports and business arenas. Specific topics covered include: Neuroscience, memory \& decision making; Behavior

modification \& management; Clinical applications; Business \& sports performance management; Social and developmental studies; Anima studies. The manuscript management system is completely online and includes a quick and fair peer-review system. Visit http://www.dovepress. com/testimonials.php to read real quotes from published authors. 